\title{
Prenatal Diagnosis of Isolated Hypospadias by Using “Tulip Sign” with Two and Three-Dimensional Ultrasonography
}

\author{
Sebahat ATAR GÜREL ${ }^{1}$
}

Ordu, Turkey

\begin{abstract}
Hypospadias is an anomaly of the male urogenital tract which occurs as a result of deficient closure of the urethral groove. Hypospadias is the most commonly seen urogenital anomaly in male neonates, but it is rarely diagnosed during prenatal period. Herein, a severe hypospadias case which was diagnosed by using 2D (two-dimensional) and 3D (three-dimensional) ultrasonography at 28 weeks of pregnancy with the aid of "tulip sign" finding was presented. 2D ultrasonography of 29 year old, primigravid woman at 28 weeks of gestation revealed an incurved, short penis with blunt tip located between the two scrotal folds and there was no additional anomaly. Findings were compatible with the "tulip sign" and case was diagnosed as isolated hypospadias. Isolated hypospadias was confirmed after delivery and surgical correction was planned. Prenatal diagnosis of hypospadias and relevant literature has been reviewed.
\end{abstract}

Keywords: Hypospadias, Ultrasonography, Prenatal diagnosis, Tulip sign

Gynecol Obstet Reprod Med 2016;22:54-56

\section{Introduction}

Hypospadias is a congenital defect of male genitalia, which is characterized by the opening of the urinary tract on the ventral side of the penis instead of the tip. It is the most commonly seen urogenital anomaly in male neonates with an estimated prevalence of $0.20 \%-0.30 \%$ in live births. ${ }^{1,2}$ However, it is rarely diagnosed during prenatal period.

Prenatal diagnosis of hypospadias with other anomalies or without has been reported before. ${ }^{3-6}$ Meizner et al. presented a specific sonographic indication of severe hypospadias and termed this finding as "tulip sign".

Although ultrasonographic prenatal diagnosis of hypospadias is known for a long time and hypospadias associated with other anomalies are generally diagnosed prenatally without difficulty, there are a few case reports about prenatal diagno-

\footnotetext{
${ }^{1}$ Department of Obstetrics and Gynecology Faculty of Medicine Ordu University, Ordu

Address of Correspondence: Sebahat Atar Gürel

Department of Gynecology and

Obstetrics, Medical School Medipol

University, Istanbul, Turkey

sebhul@yahoo.com

Submitted for Publication: 24. 10. 2014

Accepted for Publication: 28. 11. 2014
}

«:This study has been presented as a poster at The World Congress on Building Consensus out of Controversies in Gynecology, Infertility and Perinatology (BCGIP.COGI) meeting in Istanbul, May 2013, Turkey. sis of isolated hypospadias cases. We presented a severe hypospadias case which was diagnosed by detecting "tulip sign" at 28 weeks of pregnancy by using two and three dimensional ultrasonography. Despite the fact that fetal gender determination is done almost routinely in Turkey, this is the first prenatal diagnosis of isolated hypospadias case as far as is known in the country. Also, it is one of the few case reports about prenatal diagnosis of isolated hypospadias by using "tulip sign" finding after it was described by Meizner et al. ${ }^{7}$

\section{Case Report}

A 29 year old, primigravid, healthy woman with no significant past medical and obstetric history was admitted for ultrasonographic evaluation at 28 weeks of gestation. Fetal growth was compatible with 28 weeks of gestation. Two-dimensional ultrasonography revealed an incurved, short penis with blunt tip located between the two scrotal folds (Figure 1A and B). There was no additional abnormality in the detailed sonographic examination of the fetus. 3D sonographic evaluation was done to better define the fetal external genitalia and to confirm the diagnosis (Figure 2). Ultrasonographic external genitalia findings resembled the "tulip sign" and the case was diagnosed as isolated hypospadias.

A male newborn weighing 2520 grams was delivered by cesarean section because of intrauterine growth restriction and fetal distress at 38 weeks. Hypospadias was confirmed after delivery and additional anomaly was not detected (Figure 3). Postnatal karyotyping revealed 46, XY. The baby is doing well and waiting for surgical correction. 

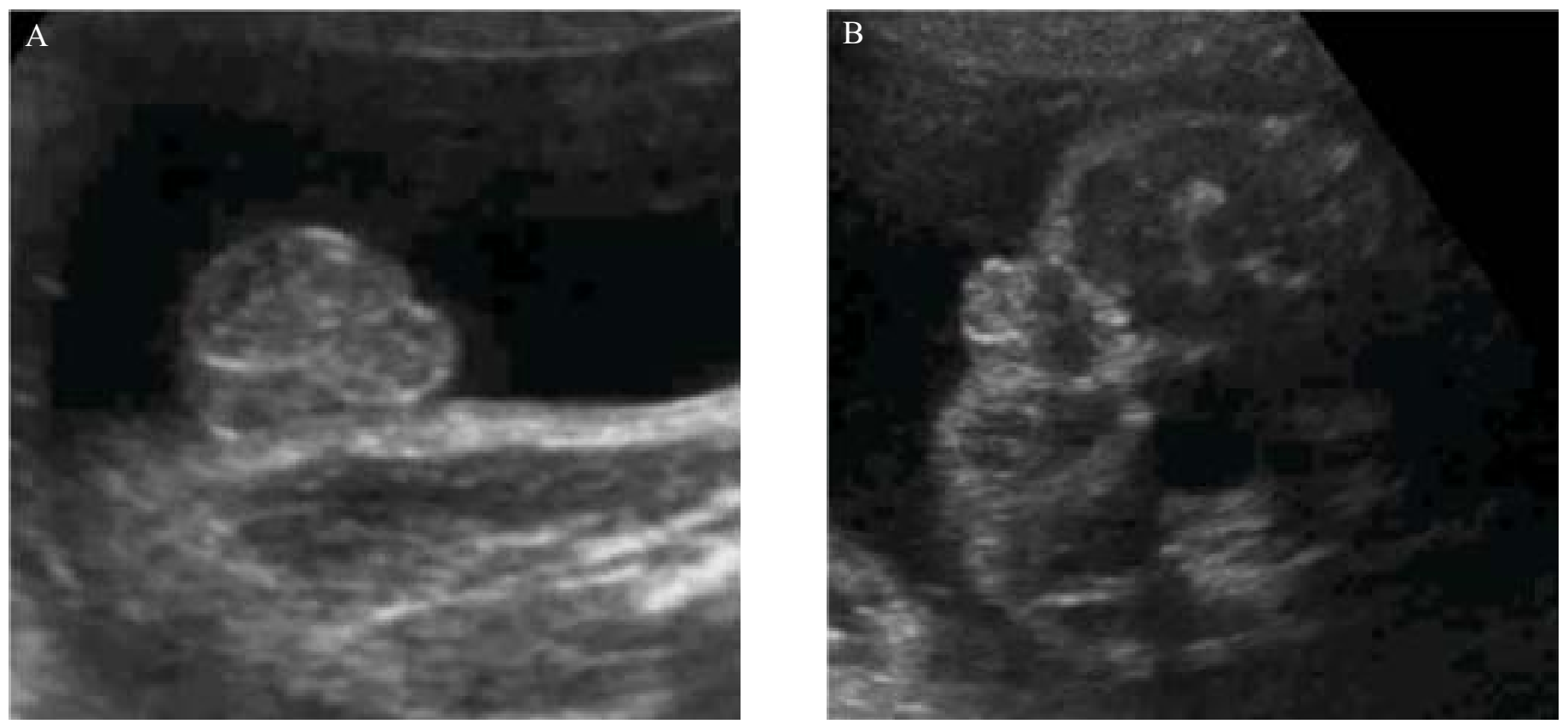

Figure 1A and B: Two dimensional ultrasound image showing the incurved, small penis with blunted tip situated between the 2 scrotal folds (tulip sign)

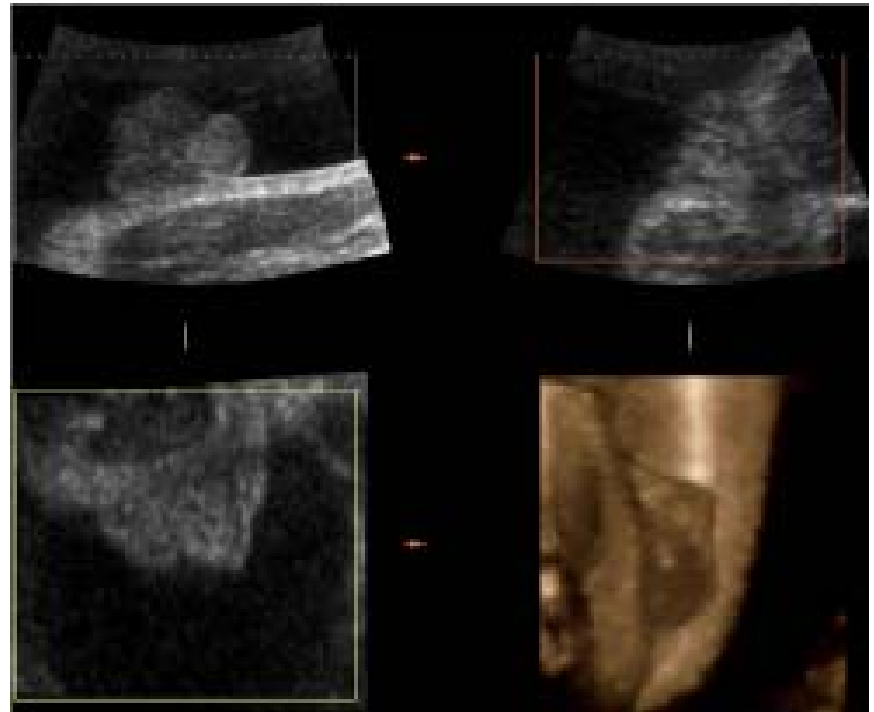

Figure 2: Thee-dimensional multiplanar images showing longitudinal (top left), coronal (top right), axial (bottom left) segment views and surface-rendered image (bottom right). "Tulip sign" is clearly seen in the longitudinal plane. Surface-rendered image shows short, incurved penis.

\section{Discussion}

Hypospadias is a developmental anomaly of the male urethra and characterized by the location of urethral meatus on the ventral side of the penis instead of the tip. Hypospadias develops as a result of a failure of closure of the urethral groove. The urethra may terminate between the proximal glans and perineum and is called as glandular, penile, penoscrotal, or perineal hypospadias according to termination site. Estimated prevalence of hypospadias is $0.20 \%-0.30 \%$ in live births. ${ }^{1,2}$ Hypospadias is seen more commonly in whites and in

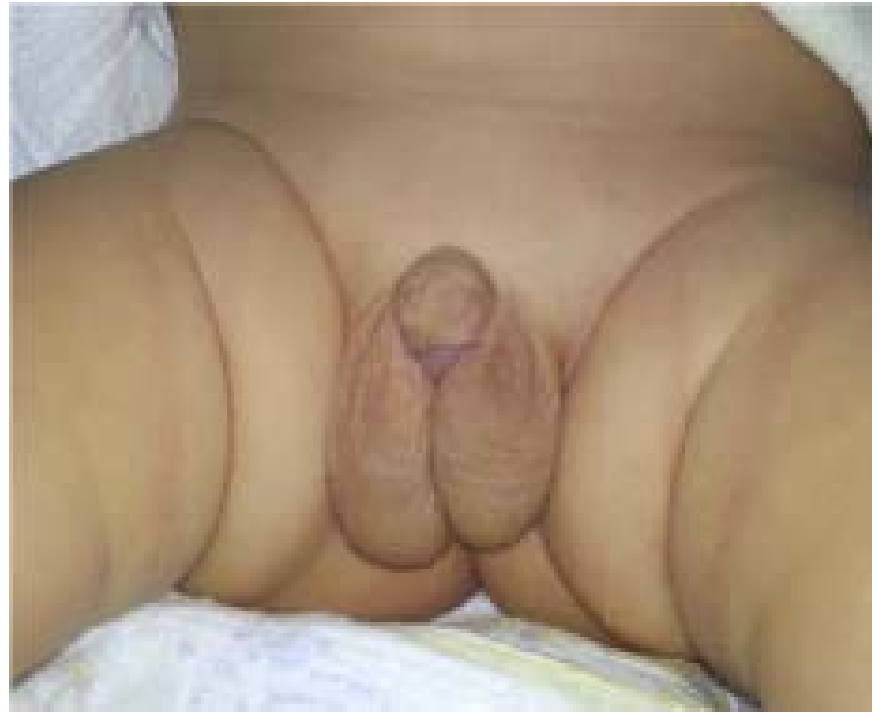

Figure 3: The postnatal appearance similar to prenatal ultrasound images and confirms the hypospadias.

monozygotic twins. ${ }^{8,9}$ Hypospadias is usually seen as an isolated abnormality. However, prenatal diagnosis of hypospadias is commonly done in cases associated with other anomalies or dysmorphic syndromes., ${ }^{4,10-12}$ Prenatal diagnosis of hypospadias is usually made in late second or third trimester of pregnancy.

Hypospadias is one of the most common urogenital anomalies, but diagnosis is often missed before birth. Ultrasonographic features of hypospadias was described before as; abnormal ventral curvature of the distal penis, small penis with ventral incurving, small lateral folds, abnormal distal morphology of the penis, abnormal fetal micturition. ${ }^{5,6,13}$ 
Meizner et al. ${ }^{7}$ presented a specific sonographic appearance of severe hypospadias and termed this finding as "tulip sign" and they gave sonographic markers for prenatal diagnosis of hypospadias as following:

- Absence of the normal pointed morphology of the penile shaft with blunted tip.

- Abnormal curvature of the penis.

- A small penile shaft.

- Two parallel echogenic lines corresponding to lateral folds of the dermal remains of the prepuce.

- Ventral deflection of urinary stream."

Three of these findings were observed by using ultrasound in this case. These findings were abnormal curvature of the penis, small penile shaft and penile shaft with blunted tip. Multiplanar and surface-rendered images of the external genitalia were acquired in the axial, coronal and midsagittal planes (Figure 2). Three dimensional ultrasound examinations in the surface rendered mode allowed us to establish more distinct appearance of external genitalia and to confirm the diagnosis of hypospadias. According to Meizner et al. " "tulip sign" represents the most severe form of hypospadias. Therefore; they proposed that the "tulip sign" may help to distinguish between severe hypospadias and other forms of genital abnormalities. When hypospadias repair surgery is performed at an early stage, the cosmetic and functional results are excellent. Therefore, correct diagnosis of severe hypospadias by using the "tulip sign" will reassure the parent especially in ambiguous genitalia cases.

Only one publication could be found about prenatal diagnosis of hypospadias in our country. In this publication, Aslan et al. ${ }^{3}$ reported prenatal diagnosis of Wolf-Hirschhorn syndrome (4p-) in association with congenital hypospadias and foot deformity. Ultrasonographic appearance of hypospadias was defined as "irregular distal penis" in this case report. In our country, where families are very curious about the gender of fetus, fetal gender determination is done almost routinely during prenatal ultrasound examination. It is very interesting that despite almost routine fetal gender determination, this is the first prenatal diagnosis of isolated hypospadias case as far as is known in our country. Also, only one case report could be found about "tulip sign" and prenatal diagnosis of hypospadias by ultrasonography in the literature. ${ }^{14}$ The most probable reason for this is that ultrasonography of genitalia include sex determination but not anomaly detection. According to Devesa et al. ${ }^{6}$; the purpose of prenatal evaluation of external genitalia should not be only to determine the sex, but also to detect anomalies. Therefore, targeted ultrasound should include fetal sex determination and external genitalia evaluation of anomalies.

Hypospadias may be associated by some syndromes and chromosome abnormalities. Therefore, prenatal diagnosis is important to plan karyotyping, to look for association with any possible dysmorphic syndromes and to reassure the parents. Three dimensional ultrasound may improve the diagnostic ac- curacy by enabling direct visualization of hypospadias and should be used whenever possible. Prenatal ultrasound should include not only fetal sex determination but also detection of abnormal genitalia.

\section{References}

1. Andersen B, Mitchell M. Recent advances in hypospadias: current surgical technique and research in incidence and etiology. Curr Urol Rep 2001;2:122-6.

2. Fujimoto T, Suwa T, Kabe K, Adachi T, Nakabayashi M, Amamiya T. Placental insufficiency in early gestation is associated with hypospadias. J Pediatr Surg 2008;43:358-61.

3. Aslan H, Karaca N, Basaran S, Ermis H, Ceylan Y. Prenatal diagnosis of Wolf-Hirschhorn syndrome (4p-) in association with congenital hypospadias and foot deformity. BMC Pregnancy and Childbirth 2003;3:1-6.

4. Tonni G, Centini G, Bonasoni MP, Ventura A, Pattacini P, Cavalli P. Acrania-anencephaly associated with hypospadias. Prenatal ultrasound and MRI diagnosis and molecular folate metabolism pathway analysis. Fetal Pediatr Pathol 2012;31:379-87.

5. Sides D, Goldstein RB, Baskin L, Kleiner BC. Prenatal diagnosis of hypospadias. J Ultrasound Med 1996;15:741-6.

6. Devesa R, Muñoz A, Torrents M, Comas C, Carrera JM. Prenatal diagnosis of isolated hypospadias. Prenat Diagn 1998;18:779-88.

7. Meizner I, Mashiach R, Shalev J, Efrat Z, Feldberg D. The 'tulip sign": a sonographic clue for in-utero diagnosis of severe hypospadias. Ultrasound Obstet Gynecol 2002; 19:250-3.

8. Welch KJ. Hypospadias. In Ravitch MM, Welch KJ, Benson CD, eds. Pediatric Surgery, 3 edn. Chicago: Year Book Medical Publishers 1979;1353-76.

9. Roberts CJ, Lloyd S. Observations on the epidemiology of simple hypospadias. BMJ 1973;1:765.

10. Cerasaro TS, Brock WA, Kaplan GW. Upper urinary tract anomalies associated with congenital hypospadias: is screening necessary? J Urol 1986;135:537-42.

11. Sepulveda W. Prenatal 3-Dimensional Sonographic Depiction of the Wolf-Hirschhorn Phenotype. The "Greek Warrior Helmet" and "Tulip" Signs. J Ultrasound Med 2007;26:407-10.

12. Tajima H, Itoh H, Mochizuki A, et al. Case of polyhydramnios complicated by Opitz G/BBB syndrome. J Obstet Gynaecol Res 2010;36:876-81.

13. Smulian JC, Scorza WE, Guzman ER, Ranzini AC, Vintzileos AM. Prenatal sonographic diagnosis of mid shaft hypospadias. Prenat Diagn 1996;16:276-80.

14. Rios LT, Araujo Júnior E, Nardozza LM, et al. Prenatal diagnosis of penoscrotal hypospadia in third trimester by two- and three-dimensional ultrasonography: a case report. Case Rep Urol 2012;2012:142814. 